\title{
Was Sie aus diesem Essential mitnehmen können
}

- Die Reduzierung des Dispositionseffektes auf die populäre Formel, dass Gewinner zu früh und Verlierer zu spät verkauft werden, greift zu kurz und rückt zu stark die zeitliche Dimension in den Vordergrund. Vielmehr ist der Wesenskern des Dispositionseffektes, dass das Individuum eine Neigung verspürt Gewinne gegenüber Verlusten bevorzugt zu realisieren. Dies äußert sich nicht ausschließlich in dem zeitlichen Unterschied der Realisation.

- Der Dispositionseffekt kann nicht überzeugend durch Modelle erklärt werden, die sich an einem neoklassischen Verständnis von rational orientieren.

- Gleichwohl die alternativen Erklärungsmodelle, wie bspw. die Prospect Theory und der Seeking-Pride-Avoiding-Regret-Ansatz, mehr überzeugen, weisen sie im Spiegel der empirischen Überprüfung Schwächen auf. Nichtsdestotrotz ist die Prospect Theory in der Literatur das am häufigsten verwendete Modell, um den Dispositionseffekt zu erklären.

- Je größer die Erfahrung, je höher der formale Bildungsabschluss und der Intelligenzquotient des Investors sind, desto geringer ist der Dispositionseffekt ausgeprägt. Der Dispositionseffekt ist bei professionellen Investoren geringer als bei privaten Investoren und stellt sich auf einem Sättigungsniveau ein.

- Die Tendenz Gewinne gegenüber Verlusten bevorzugt zu realisieren, ist kulturell stabil und neben Wertpapieren auch für Immobilien dokumentiert. Zudem kann der Dispositionseffekt auch unter kontrollierten Bedingung im Experiment nachgewiesen werden. 